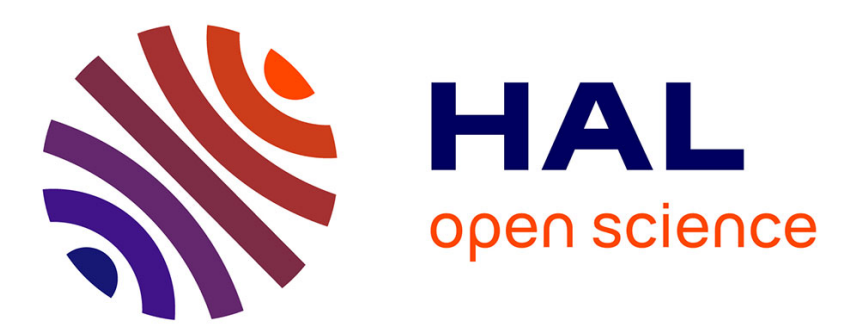

\title{
Qualitative analysis of classes of motion for multiresonant systems II. A geometrical method
}

Angelo Luongo, Angelo Di Egidio, Achille Paolone

\section{To cite this version:}

Angelo Luongo, Angelo Di Egidio, Achille Paolone. Qualitative analysis of classes of motion for multiresonant systems II. A geometrical method. Acta Mechanica, 2005, 174 (1-2), pp.109-124. hal00790811

\section{HAL Id: hal-00790811 \\ https://hal.science/hal-00790811}

Submitted on 21 Feb 2013

HAL is a multi-disciplinary open access archive for the deposit and dissemination of scientific research documents, whether they are published or not. The documents may come from teaching and research institutions in France or abroad, or from public or private research centers.
L'archive ouverte pluridisciplinaire HAL, est destinée au dépôt et à la diffusion de documents scientifiques de niveau recherche, publiés ou non, émanant des établissements d'enseignement et de recherche français ou étrangers, des laboratoires publics ou privés. 


\title{
Qualitative analysis of classes of motion for multiresonant systems II. A geometrical method
}

\author{
A. Luongo, A. Di Egidio, L'Aquila and A. Paolone, Rome, Italy
}

\begin{abstract}
Summary. Classes of motion of general multiresonant systems are derived through a geometrical algo rithm based on a set representation. First, the elementary classes existing under simple resonance condi tions are evaluated; rules governing the interaction between elementary classes belonging to different resonance conditions are then drawn up as applications of a unique theorem. Illustrative examples are given. The method also permits a hierarchical ordering of the amplitudes of the resonant modes, according to their participation in the classes; it also makes it possible to ascertain in advance the existence of a standard form for the amplitude modulation equations. The stability analysis of incomplete steady solutions is then addressed. Three classes of perturbation are distinguished, namely: in class perturbations, out of class resonant perturbations, and out of class nonresonant perturbations. The structure of the Jacobian variational matrix is studied. The Jacobian matrix is shown to comprise three diagonal blocks associated with the three perturbation classes, so that the stability equations are uncoupled. Further possible uncouplings of one of the blocks are analyzed in relation to some of the geometrical properties of the classes.
\end{abstract}

\section{Introduction}

The problem of evaluating all the classes of motion of a multiresonant system on the basis of knowledge of the resonance conditions only (i.e., before deriving the Amplitude Modulation Equations, AMEs) is addressed in Part I of this paper [1]. There, an algebraic method (the Resonance Coefficient Method, RCM) is illustrated to achieve the goal. Here, an alternative geometrical approach (the Class Diagram Method, CDM) is presented.

Although the RCM is an efficient tool to solve the problem, the CDM presents new features that make its application useful. It consists in representing the classes as sets of the space of the amplitudes. Each resonance condition entails the existence of elementary classes which are represented as a collection of sets, called families of classes. When two or more families interact (i.e., when two or more resonance conditions exist) some of the classes survive, others disappear and others merge to form a new family. The rules governing these interactions are found to descend from a unique theorem, which is proved here. The diagrams obtained provide a very comprehensive representation of all the existing classes; they also make it possible to evaluate the amplitudes that contribute most to the motion, as well as to ascertain if conditions occur for the existence of a standard form of the amplitude equations, according to a previous paper by the authors [2]. 
The stability problem is also addressed and the structure of the variational equations governing their stability is analyzed. It is shown that the Jacobian matrix is block-diagonal, each block being associated with a different class of perturbation, namely: in-class, resonant out-ofclass and non-resonant out-of-class perturbations. Further uncouplings of one of these blocks are revealed by the way the classes of motion are nested in the diagram.

\section{Classes of motion}

The problem of evaluating classes of motion is posed and some definitions are formally given. Elementary and multiple classes are then studied.

\subsection{Statement of the problem and definitions}

A multiresonant (continuous or discrete) nonlinear system is given, in which $N$ natural frequencies $\omega_{n}$ and (possibly) a forcing frequency $\omega_{0}$ are involved in $S$ resonance conditions

$\sum_{n \in \mathcal{N}^{+}} k_{s n} \omega_{n}=0 \quad s=1,2, \ldots, S \quad k_{s n} \in \mathbb{Z}$,

where $\mathscr{N}^{+}=\{0,1, \ldots, N\}$ and small detunings have been neglected. Among Eqs. (1), $R:=\operatorname{rank}\left[k_{s n}\right]$ are independent equations, while the remaining $Q:=S-R$ are linear combinations of the former.

The nonlinear asymptotic behavior of the system is described by the evolution in time of $N$ complex quantities $A_{n}(t)$; these are the amplitudes of the $N$ interacting linear modes of frequencies $\omega_{n}$. If the system is forced, the AMEs also depend on the amplitude $A_{0}$ of the excitation. The evolution is governed by the so-called Amplitude Modulation Equations (AMEs), as, e.g., furnished by the Multiple Scale Method (MSM). These equations, whose structure has been studied in depth in [1], admit solutions in which only one subset of amplitudes is different from zero, while the remaining amplitudes identically vanish. Such solutions have been named (incomplete) classes of motion, or $M$-(modal) classes, i.e., classes in which only $M$-amplitudes contribute to the evolution. The question analyzed here consists in evaluating all the classes of motion, without deriving the AMEs in advance, simply by using Eqs. (1). An algebraic method to address the problem is illustrated in [1] (Resonance Coefficient Method, RCM); here, a geometrical approach is followed instead, based on a set representation of the classes. Some definitions are preliminarily given.

The set $\mathscr{S}:=\left\{A_{n} \mid n \in \mathscr{N}^{+}\right\}$of all the amplitudes involved in the $S$ resonances (1) is called the state-space of the dynamical system $A_{m}^{\prime}=\mathscr{L}_{m}(\mathscr{S})$ (AMEs). The set $\mathscr{S}_{S} \subseteq \mathscr{S}$ of the amplitudes $A_{n}$ associated with the frequencies $\omega_{n}$ involved in the s-th resonance is called the elementary s-th space, or the $s$-th resonance space; by remembering Eqs. (1) it is $\mathscr{S}_{S}:=\left\{A_{n} \mid k_{s n} \neq 0\right\}$. A set $\mathscr{C}=\left\{A_{i}, i \in \mathscr{M}:=\left\{i_{1}, i_{2}, \ldots, i_{M}\right\}\right\}$ is an M-class of motion for the system if it is anvariant subspace of $\mathscr{S}$; this property holds if and only if $\mathscr{L}_{j}(\mathscr{S}) \equiv 0 \forall j \notin \mathscr{M}$, for any $A_{i} \in \mathscr{C}$. If the forcing amplitude $A_{0} \in \mathscr{S}$, then necessarily $A_{0} \in \mathscr{C}$, since it cannot vanish. As particular cases, the null set $\{0\}$ and the state space $\mathscr{S}$ are classes of motion, containing no components or all the components, respectively. The classes of motion admitted by the $s$-th resonance (i.e., as invariant subspaces of $\mathscr{S}_{S}$ ) are called the elementary classes of motion $\mathscr{C}_{S_{1}}, \mathscr{C}_{S_{2}}, \ldots$ of the $s$-th resonance. The set of all the elementary classes of motion $\mathscr{C}_{S_{i}}$ is called the family of classes of motion of the $s$-th resonance $\mathscr{F}_{s}:=\left\{\mathscr{C}_{S_{1}}, \mathscr{C}_{S_{2}}, \ldots\right\}$; the family contains the null-set. The classes 
of motion admitted by two (or more) resonances, e.g., the $r$-th and the $s$-th, are the invariant subspaces of $\mathscr{S}_{r} \cup \mathscr{S}_{s}$; they are referred to as multiple classes of motion $\mathscr{C}_{r s_{1}}, \mathscr{C}_{r s_{2}}, \ldots$, and their collection as the multiple family $\mathscr{F}_{r s}$.

The algorithm described below first calls for evaluation of the elementary classes of motion of each resonance, and then for their combination in multiple classes.

\subsection{Elementary classes}

The classes of motion existing under the most common simple $(S=1)$ resonance conditions are analyzed. To evaluate them, reference can be made to the relevant AMEs, listed in Tables 1 and 2 of [1]. For example, if the resonance $\omega_{i}=2 \omega_{j}$ is considered, the system equations read (by retaining only the essential terms) $A_{i}^{\prime}=\mathscr{L}_{i}\left(A_{j}^{2}\right), A_{j}^{\prime}=\mathscr{L}_{j}\left(A_{i} \overline{A_{j}}\right)$. It follows that while $\mathscr{C}_{1}:=\left\{A_{i}\right\}$ is a 1 -class (since $A_{j}=0$ entails $\mathscr{L}_{j}=0$ ), $\left\{A_{j}\right\}$ is not (since $\mathscr{L}_{i} \neq 0$ when $A_{i}=0$ ). Moreover, $\mathscr{C}_{2}:=\left\{A_{i}, A_{j}\right\}$ is a 2-class, since it coincides with the space $\mathscr{S}_{S}$ of the resonance (complete class). The two elementary classes constitute the family $\mathscr{F}_{s}:=\left\{\mathscr{C}_{1}, \mathscr{C}_{2}\right\}$ associated with the resonance $\omega_{i}=2 \omega_{j}$. The same result is achieved in a simpler way if the RCM [1] is applied: since $k_{s i}=1, k_{s j}=-2,\left\{A_{i}\right\}$ is admitted (since $\left|k_{s j}\right| \neq 1$ ) while $\left\{A_{j}\right\}$ is forbidden (since $\left.\left|k_{s i}\right|=1\right)$.

An efficient geometrical representation of the elementary classes of motion is given in Tables 1 to 3 . The classes are represented by closed curves (sets) surrounding the amplitudes. Table 1 illustrates the internal (not forced) quadratic (order $K=2$ ) and cubic (order $K=1$ and 3 ) resonances. In the $\omega_{i}=2 \omega_{j}$ case previously discussed only a monomodal solution exists. In the quadratic combination resonance $\omega_{i}=\omega_{j} \pm \omega_{k}$, the three 1-class solutions are all possible; however, no bimodal motion exists, since two amplitudes different from zero would trigger the third amplitude. On proceeding to analyze the cubic reso-

Table 1. Elementary classes of motion

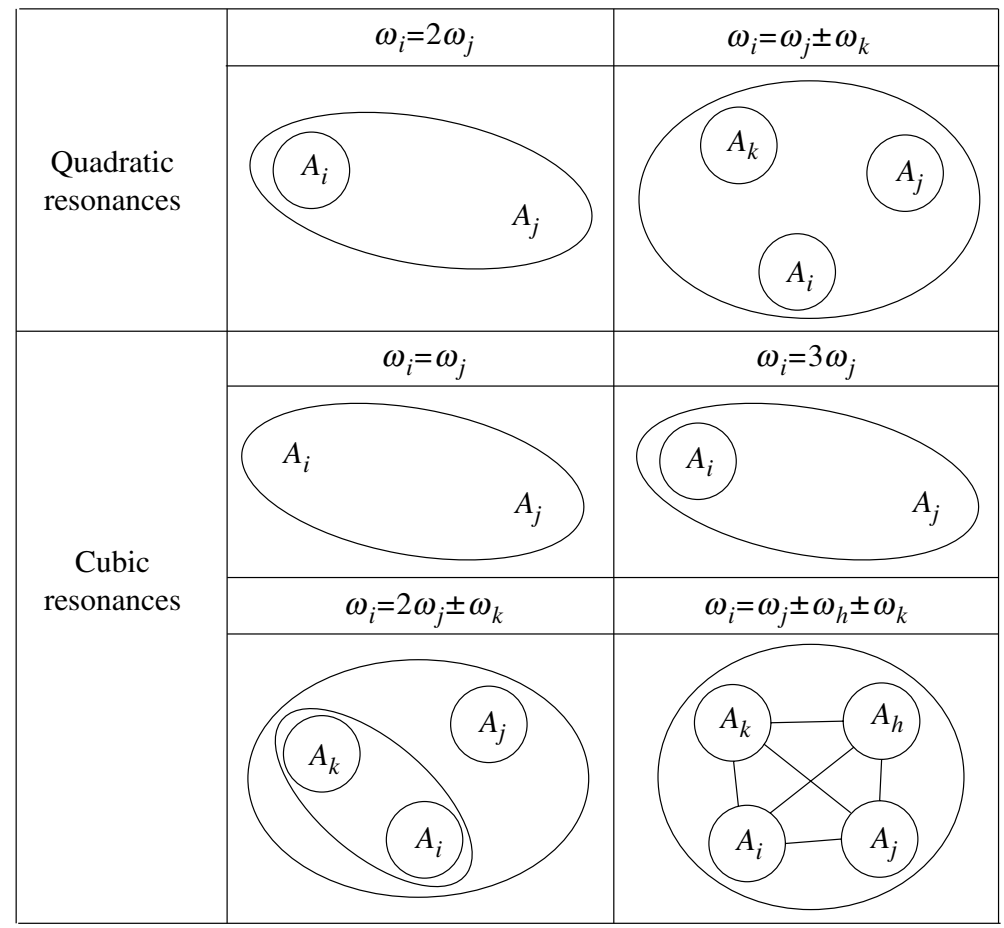


Table 2. Special cases of elementary classes of motion in the $\omega_{i}=\omega_{j}$ resonance

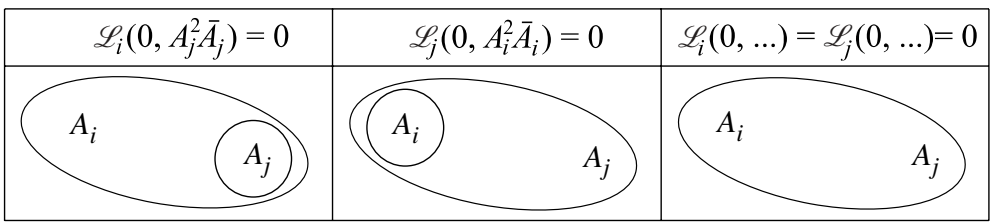

nances, in the $\omega_{i}=\omega_{j}$ case no incomplete motions are found. In the $\omega_{i}=3 \omega_{j}$ resonance, the monomodal of highest frequency is admitted, similarly to the quadratic case. The $\omega_{i}=2 \omega_{j} \pm \omega_{k}$ resonance is also similar to the quadratic combination resonance, since all the monomodal solutions are allowed; however, in addition to that, a bimodal motion $\left\{A_{i}, A_{k}\right\}$ exists. Finally, in the other cubic combination resonance, $\omega_{i}=\omega_{j} \pm \omega_{h} \pm \omega_{k}$, in addition to all the monomodal motions, all bimodal solutions exist (represented in the figure by solid lines, for the sake of clarity); however, no trimodal motions are allowed, since no amplitude can vanish alone.

The above results refer to the generic case in which all the coefficients of the AMEs are different from zero. However, if some of them vanish, a richer scenario exists. To illustrate this behavior the $\omega_{i}=\omega_{j}$ resonance is considered again as an example. The essential (primary resonant) terms of the relevant AMEs read (see Table 1 of [1]) $A_{i}^{\prime}=\mathscr{L}_{i}\left(A_{i} \overline{A_{i}} A_{j}, A_{j}^{2} \overline{A_{j}}, A_{j}^{2} \overline{A_{i}}\right), A_{j}^{\prime}=\mathscr{L}_{j}\left(A_{j} \overline{A_{j}} A_{i}, A_{i}^{2} \overline{A_{i}}, A_{i}^{2} \overline{A_{j}}\right)$. Therefore, the relevant $A_{i}$ - (or $A_{j}$-)equation contains terms that are pure in $A_{j}$ (or $A_{i}$ ) and which prevent the occurrence of monomodal solutions. However, if one or both of these terms have a zero coefficient, one or both monomodal solutions do exist (see Table 2). One remarkable mechanical system exhibiting such behavior is the $3 \mathrm{D}$ beam of a compact section in 1:1 internal resonance, which in fact possesses two monomodal solutions, each consisting of a flexural vibration in a principal inertia plane [3].

The external (or mixed) resonance conditions are now considered. By examining the AMEs in Table 2 of [1], or applying the RCM, the classes of motion depicted in Table 3 are found.

Table 3. Elementary classes of motion of forced systems

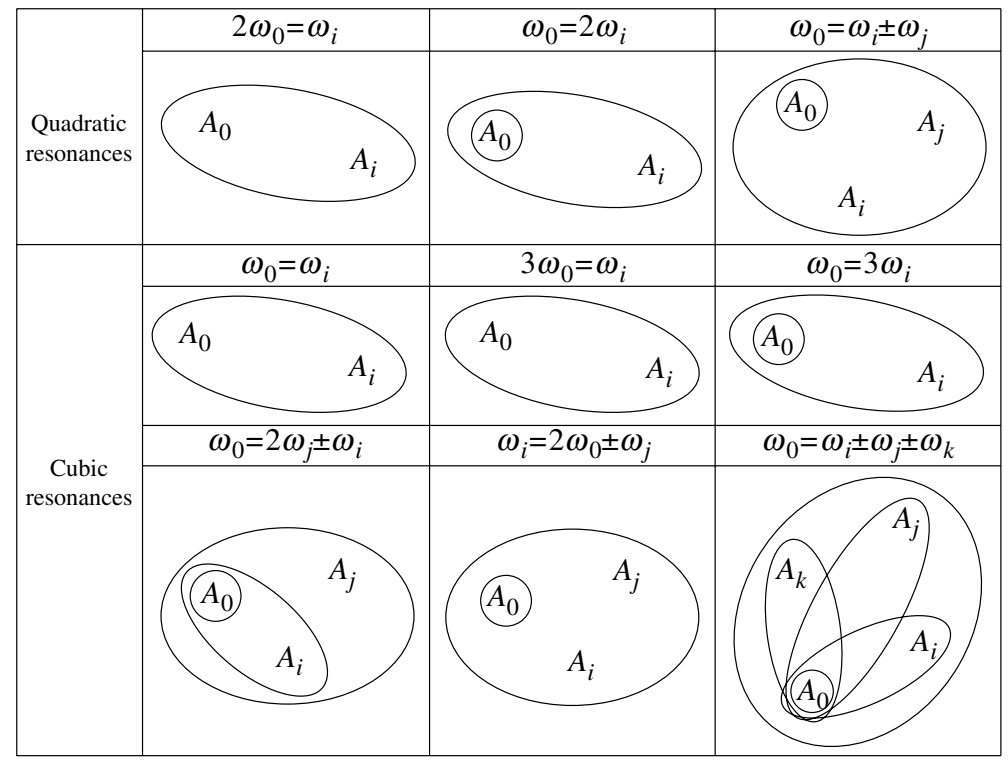


These can also be obtained from Table 1 by identifying $\omega_{0}$ with a natural frequency and remembering that the externally resonant classes must contain $A_{0}$. Therefore, in the superharmonic resonances $\omega_{i}=k \omega_{0}(k=2,3)$ the highest frequency monomodal solution disappears, while in the sub-harmonic resonances $\omega_{0}=k \omega_{i}(k=2,3)$ the highest 1-class $\left\{A_{0}\right\}$ is admitted. This describes a motion in which the $i$-th modal coordinate is simply forced by the external excitation (particular solution of the differential equation) but no free oscillations of amplitude $A_{i}$ are triggered (homogeneous solution). The resonance $\omega_{0}=\omega_{i} \pm \omega_{j}$ can be formally obtained from the $\omega_{i}=\omega_{j} \pm \omega_{k}$ internal resonance by replacing one of the amplitudes with $A_{0}$. Among the monomodal solutions, only $\left\{A_{0}\right\}$ survives; since no bimodal solutions exist in the unforced case, no other classes are admitted in addition to the complete one. By a similar procedure the resonances $\omega_{0}=2 \omega_{j} \pm \omega_{i}$ and $\omega_{i}=2 \omega_{0} \pm \omega_{j}$ can be derived from the unforced $\omega_{i}=2 \omega_{j} \pm \omega_{k}$ case. Among the monomodal solutions, again only $\left\{A_{0}\right\}$ survives; moreover, the bimodal solution of the unforced case persists only if $\omega_{0}$ is identified as $\omega_{k}$ or $\omega_{i}$. Finally, the $\omega_{0}=\omega_{i} \pm \omega_{j} \pm \omega_{k}$ case can be derived from the $\omega_{i}=\omega_{j} \pm \omega_{h} \pm \omega_{k}$ resonance. Only the monomodal solution $\left\{A_{0}\right\}$ and all the bimodal solutions which include $A_{0}$ are classes of motion; the other bimodal solutions are destroyed instead.

\subsection{Multiple classes}

The general case of multiple $(S>1)$ resonance is now analyzed. Each of the $S$ resonance conditions (1) entails the existence of some elementary classes of motion, according to the results previously discussed. The question is to evaluate how these elementary classes interact, in order to furnish new multiple classes. To solve the problem, the following strategy is adopted. First, the interaction between two families of classes of motion, $\mathscr{F}_{1}$ and $\mathscr{F}_{2}$, associated with $s=1$ and $s=2$, respectively, is studied and a new (multiple) family, $\mathscr{F}_{12}$, is determined. The latter is combined with the $\mathscr{F}_{3}$-family associated with $s=3$ and the multiple family is updated to $\mathscr{F}_{123}$. The process is stopped when the last family $s=S$ has been considered, namely $\mathscr{F}_{12 \ldots . . S}$. The task thus essentially consists in analyzing the simplest problem $S=2$. Reference will be initially made to internally resonant systems; the effects of the excitation will be taken into account later.

Let us consider two families $\mathscr{F}_{A}=\left\{\mathscr{C}_{A_{1}}, \mathscr{C}_{A_{2}}, \ldots\right\}$ and $\mathscr{F}_{B}=\left\{\mathscr{C}_{B_{1}}, \mathscr{C}_{B_{2}}, \ldots\right\}$, each associated with an internal resonance condition. Let $\mathscr{A}$ and $\mathscr{B}$ be the spaces of the two resonances. Moreover, let us denote by $\mathscr{W}:=\mathscr{A} \cap \mathscr{B}, \mathscr{U}:=\mathscr{A} \backslash \mathscr{W}$ and $\mathscr{V}:=\mathscr{B} \backslash \mathscr{W}$ the subsets of $\mathscr{A} \cup \mathscr{B}$ so that $\mathscr{A}=\mathscr{U} \cup \mathscr{W}$ and $\mathscr{B}=\mathscr{V} \cup \mathscr{W}$ (Fig. 1a). The conditions under which $\mathscr{C}_{A B}$ (Fig. 1b) is a multiple class of the $(\mathscr{A}, \mathscr{B})$-resonances are stated by the following theorem:

Theorem 1: Given two families of unforced classes of motion, $\mathscr{F}_{A}$ and $\mathscr{F}_{B}$, of spaces $\mathscr{A}$ and $\mathscr{B}$, respectively, a subset $\mathscr{C}_{A B}$ of $\mathscr{A} \cup \mathscr{B}$ is a class of the multiple $\mathscr{F}_{A B}$ family if and only if $\mathscr{C}_{A B} \cap \mathscr{A}$ is an elementary class of $\mathscr{F}_{A}$ and $\mathscr{C}_{A B} \cap \mathscr{B}$ is an elementary class of $\mathscr{F}_{B}$.

Mathematical proof of Theorem 1 is given in Appendix A; here it is justified by invoking mechanical concepts. Let us denote by $\widehat{\mathscr{U}}, \widehat{\mathscr{V}}$ and $\widehat{\mathscr{W}}$ the intersections of $\mathscr{C}_{A B}$ with $\mathscr{U}, \mathscr{V}$ and $\mathscr{W}$, respectively, and by $\mathscr{U}_{0}, \mathscr{V}_{0}$ and $\mathscr{W}_{0}$ their complements (see Fig. $1 \mathrm{~b}$ ), so that $\mathscr{C}_{A B} \cap \mathscr{A} \equiv \widehat{\mathscr{U}} \cup \widehat{\mathscr{W}}$ and $\mathscr{C}_{A B} \cap \mathscr{B} \equiv \widehat{\mathscr{V}} \cup \widehat{\mathscr{W}}$. It can been seen that the law of assembly of the AMEs discussed in [1] is equal to that of mechanical systems connected in series. Based on this analogy, the system in Fig. 1a is assimilated to a structure consisting of two substructures, whose displacements are $\mathscr{U} \cup \mathscr{W}$ and $\mathscr{V} \cup \mathscr{W}$, respectively. The two substructures therefore interact through the displacements at the interface $\mathscr{W}$. Let us assume that there exist classes of 


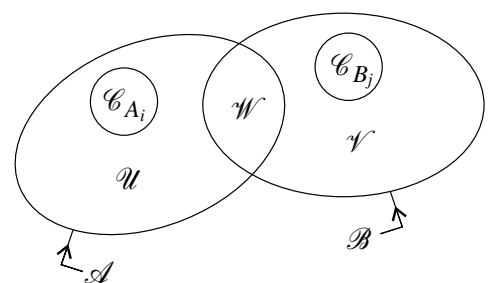

a

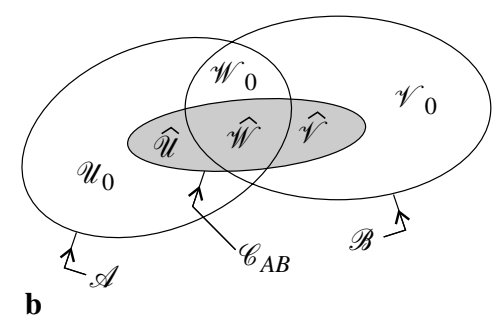

b

Fig. 1. Interaction between two families of elementary classes of motion: a dominions $(\mathscr{A}, \mathscr{B})$, sub sets $(\mathscr{U}, \mathscr{V}, \mathscr{W})$ and elementary classes $\left(\mathscr{C}_{A_{i}}, \mathscr{C}_{B_{j}}\right) ; \mathbf{b}$ multiple class $\mathscr{C}_{A B}$

motion in which the two isolated substructures oscillate in a smaller set of (arbitrary) displacements, namely $\widehat{\mathscr{U}} \cup \widehat{\mathscr{W}}$ and $\widehat{\mathscr{V}} \cup \widehat{\mathscr{W}}$, respectively (Fig. 1b). If the two structures are connected, compatibility is ensured by requiring that the (arbitrary) displacements $\widehat{\mathscr{W}}$ be equal, since the displacements $\mathscr{W}_{0}$ are zero. The incomplete motion thus also exists for the composed structure. If, in contrast, the displacements $\widehat{\mathscr{U}} \cup \widehat{\mathscr{W}}$ and/or $\widehat{\mathscr{V}} \cup \widehat{\mathscr{W}}$ are not a class of motion for the isolated substructures, they also trigger the displacements $\mathscr{U}_{0}, \mathscr{V}_{0}$ and $\mathscr{W}_{0}$, hence $\mathscr{C}_{A B}$ is not a class of motion for the composed system.

An obvious consequence of Theorem 1 is given by the following

Corollary 1: A multiple class of motion is either an elementary class or is the union of elementary classes.

Application of Theorem 1 to a number of frequently occurring cases enables us to state the results collected in Table 4, commented below. Cases I-III refer to the interactions between families not admitting incomplete motions. (I) If $\mathscr{A}$ and $\mathscr{B}$ are disjoint, then $\mathscr{A}$ is a multiple class and $\mathscr{A} \cap \mathscr{B}=\{0\} \in \mathscr{F}_{B}$, since $\mathscr{A} \cap \mathscr{A}=\mathscr{A} \in \mathscr{F}_{A}$; similar arguments hold for $\mathscr{B}$. Obviously, also $\mathscr{A} \cup \mathscr{B}$ is a multiple class. (II) If $\mathscr{A}$ and $\mathscr{B}$ have a non-trivial intersection, then neither $\mathscr{A}$ nor $\mathscr{B}$ are multiple classes, since $\mathscr{A} \cap \mathscr{B}$ is not an elementary class of either family;

Table 4. Examples of multiple classes

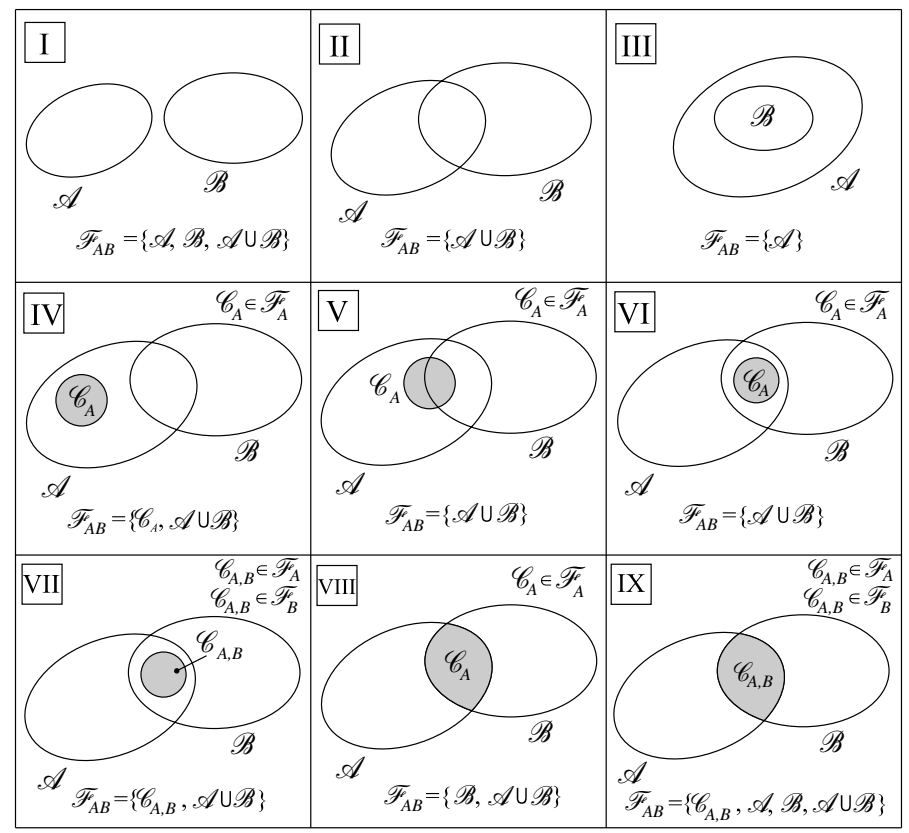


however, $\mathscr{A} \cup \mathscr{B}$ is a multiple class. (III) If $\mathscr{B} \subseteq \mathscr{A}$, since $\mathscr{B} \cap \mathscr{A}=\mathscr{B}$ is not an elementary class of $\mathscr{F}_{A}, \mathscr{B}$ is not a multiple class; in contrast $\mathscr{A} \in \mathscr{F}_{A B}$. Cases IV-VII refer to families admitting incomplete classes. In all cases $\mathscr{A} \cup \mathscr{B}$ is a multiple class; in addition, the elementary class $\mathscr{C}_{A}$ (or $\mathscr{C}_{B}$ ) is also a multiple class if it has null intersection with $\mathscr{B}$ (or $\mathscr{A}$ ) (case IV) or is contained in $\mathscr{A} \cap \mathscr{B}$ and is a class both for $\mathscr{F}_{A}$ and $\mathscr{F}_{B}$ (case VII). In contrast, $\mathscr{C}_{A}$ (or $\mathscr{C}_{B}$ ) is not a multiple class if it has non-null intersection with $\mathscr{B}$ (or $\mathscr{A}$ ) (case V) or is contained in $\mathscr{A} \cap \mathscr{B}$ but is an elementary class for only one of the two families (case VI). Finally, cases VIII and IX refer to intersections $\mathscr{A} \cap \mathscr{B}$ which are themselves elementary classes for one of the two families, or for both of them. In both cases $\mathscr{A} \cup \mathscr{B}$ is a multiple class. If $\mathscr{C}_{A} \in \mathscr{F}_{A}$ (case VIII), $\mathscr{C}_{A}$ is not a multiple class, similar to case VI; analogously, since $\mathscr{A} \cap \mathscr{B}=\mathscr{C}_{A} \notin \mathscr{F}_{B}, \mathscr{A}$ is not a multiple class; in contrast, since $\mathscr{B} \cap \mathscr{A}=\mathscr{C}_{A} \in \mathscr{F}_{A}, \mathscr{B}$ is a multiple class. Similar arguments applied to the case IX lead to the result in which $\mathscr{C}_{A B}, \mathscr{A}$ and $\mathscr{B}$ are all multiple classes.

When an external excitation acts on the system, Theorem 1 must be slightly modified. According to the results of Sect. 2.3, forced classes of motion necessarily contain the forcing amplitude $A_{0}$; therefore classes satisfying the conditions of Theorem 1 but not containing $A_{0}$ are not forced multiple classes. This is stated in the following

Corollary 2: If one or both the $\mathscr{F}_{A}$ and $\mathscr{F}_{B}$ families of Theorem 1 are associated with an external resonance condition, then the subset $\mathscr{C}_{A B}$ is a forced multiple class if and only if the conditions of Theorem 1 are satisfied and the excitation amplitude $A_{0}$ is contained in $\mathscr{C}_{A B}$.

The procedure previously illustrated is summarized by the following

Algorithm 1: CDM (Class Diagram Method): (1) the elementary class diagrams $\mathscr{F}_{i}$ of each resonance condition are drawn from Tables 1 and 3; (2) multiple class diagrams are built up by composing in sequence diagrams $\mathscr{F}_{12}, \mathscr{F}_{123}, \ldots, \mathscr{F}_{12 \ldots S}$. To compose two diagrams $\mathscr{F}_{A}$ and $\mathscr{F}_{B}$, the following strategy is adopted. According to Corollary 1, (a) the individual classes are considered and either Theorem 1 or its Corollary 2 is applied to verify whether they still survive as multiple classes, and (b) the possible merging of some classes of $\mathscr{F}_{A}$ and $\mathscr{F}_{B}$ is checked by analyzing all the combinations among the classes.

The examples of the next section illustrate the procedures.

\section{Illustrative examples}

Some examples are worked out to illustrate the geometrical method proposed. The concept of hierarchic order among the amplitudes is then introduced and the existence of standard normal form AMEs is discussed.

\subsection{Class of motion diagrams}

Let us consider a system in which $N=5$ frequencies are involved in $R=3$ independent internal resonant conditions, namely [1]:

$\omega_{1}=2 \omega_{2}, \quad \omega_{2}=\omega_{3}+2 \omega_{5}, \quad \omega_{3}=\omega_{4}$.

From Eq. (2) and $Q=1$ a dependent condition follows up to order $K=3$, namely,

$\omega_{2}=\omega_{4}+2 \omega_{5}$

The spaces of the resonances (2) are $\mathscr{S}_{1}=\left\{A_{1}, A_{2}\right\}, \quad \mathscr{S}_{2}=\left\{A_{2}, A_{3}, A_{5}\right\}$, and $\mathscr{S}_{4}=\left\{A_{3}, A_{4}\right\}$, while for the resonance (3) it is $\mathscr{S}_{3}=\left\{A_{2}, A_{4}, A_{5}\right\}$. The $\mathscr{F}_{r}(r=1,2,3,4)$ 


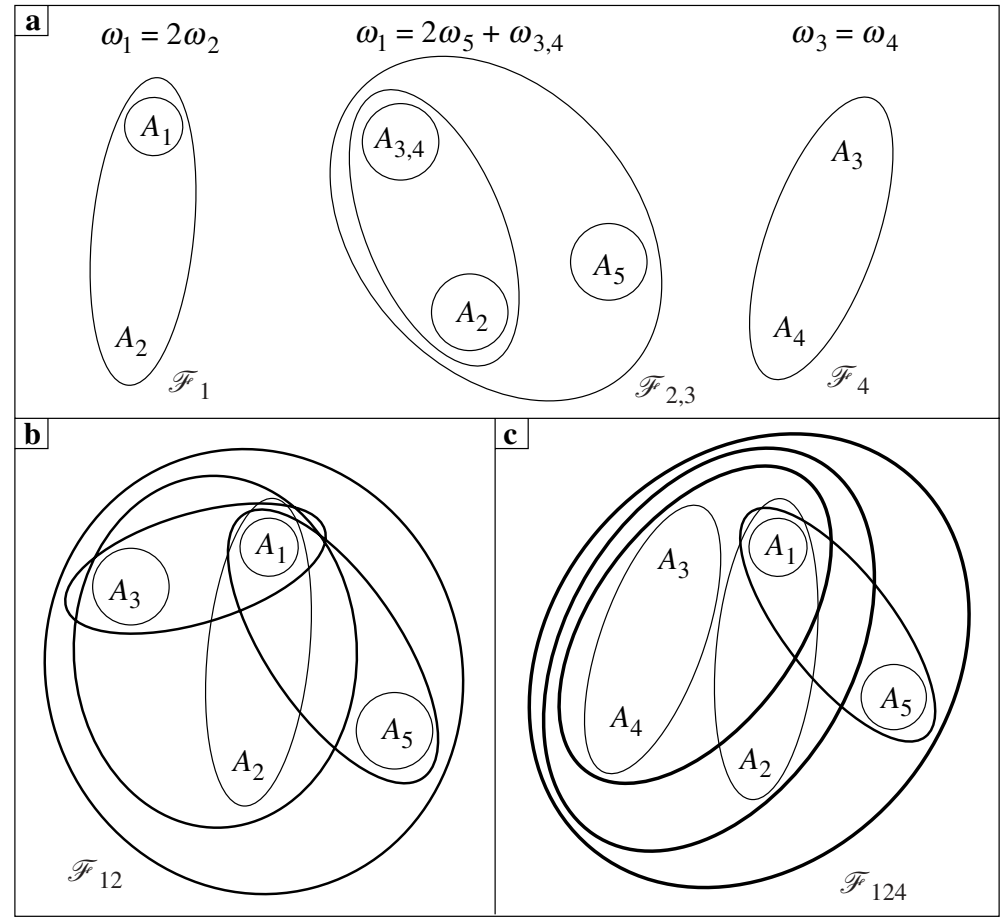

Fig. 2. Class of motion diagram for a system undergoing $R=3$ internal resonance conditions: a elementary families $\mathscr{F}_{r}(r=1,2,3,4)$ of dominions $\mathscr{D}_{r} ; \mathbf{b} \mathscr{F}_{12}$ multiple family, resulting from the interaction between $\mathscr{F}_{1}$ and $\mathscr{F}_{2} ; \mathbf{c}$ overall multiple family $\mathscr{F}_{124}$

families of elementary classes are obtained from Table 1 and are drawn in Fig. 2a in thin lines. Initially, condition Eq. (3) is ignored. First, the interaction between the $\mathscr{F}_{1}$ and $\mathscr{F}_{2}$ families is studied and the multiple family $\mathscr{F}_{12}$ (Fig. 2b) is built up as follows. The elementary monomodal solutions $\left\{A_{1}\right\},\left\{A_{3}\right\}$ and $\left\{A_{5}\right\}$ all survive to the interaction (case IV in Table 4) since they are external to $\mathscr{S}_{1} \cap \mathscr{S}_{2}=\left\{A_{2}\right\}$; in contrast, the monomodal solution $\left\{A_{2}\right\}$ disappears (case VIII) since it is not a class of $\mathscr{F}_{1}$. Similarly, the set $\left\{A_{1}, A_{2}\right\}$ is a multiple class of $\mathscr{F}_{12}$ while the sets $\left\{A_{2}, A_{3}\right\}$ as well as $\left\{A_{2}, A_{3}, A_{5}\right\}$ are not. To check if other classes emerge from the interaction, combinations between the elementary classes must be considered. Thus, two new bimodal solutions $\left\{A_{1}, A_{3}\right\}$ and $\left\{A_{1}, A_{5}\right\}$, a new trimodal solution $\left\{A_{1}, A_{2}, A_{3}\right\}$ and, finally, a new four-modal solution $\left\{A_{1}, A_{2}, A_{3}, A_{5}\right\}$ are found to be multiple classes. They are drawn in Fig. $2 \mathrm{~b}$ as thicker curves. As a second step, the interaction between the $\mathscr{F}_{4}$ - and $\mathscr{F}_{12}$-families is studied and the new $\mathscr{F}_{124}$-family (Fig. 2c) is built up as follows. Among the monomodal solutions, $\left\{A_{1}\right\}$ and $\left\{A_{5}\right\}$ survive (case IV), while $\left\{A_{3}\right\}$ disappears (case VIII). Among the bimodal solutions, $\left\{A_{1}, A_{2}\right\},\left\{A_{1}, A_{5}\right\}$ and $\left\{A_{3}, A_{4}\right\}$ still exist, while $\left\{A_{1}, A_{3}\right\}$ is destroyed. Similarly, $\left\{A_{1}, A_{2}, A_{3}\right\}$ and $\left\{A_{1}, A_{2}, A_{3}, A_{5}\right\}$ disappear. New classes, however, are formed by merging some elementary classes of $\mathscr{F}_{12}$ and $\mathscr{F}_{4}$, namely: $\left\{A_{1}, A_{3}, A_{4}\right\},\left\{A_{1}, A_{2}, A_{3}, A_{4}\right\}$ and $\left\{A_{1}, A_{2}, A_{3}, A_{4}, A_{5}\right\}$, drawn in heavy type in Fig. 2c, which all satisfy Theorem 1 . In contrast, $\left\{A_{3}, A_{4}, A_{5}\right\}$ and $\left\{A_{1}, A_{3}, A_{4}, A_{5}\right\}$ are not multiple classes, since their intersection with $\mathscr{S}_{12}=\mathscr{S}_{1} \cup \mathscr{S}_{2}$, i.e., $\left\{A_{3}, A_{5}\right\}$ and $\left\{A_{1}, A_{3}, A_{5}\right\}$, are not elementary classes of $\mathscr{F}_{12}$. The last graph thus obtained (Fig. 2c) represents all the classes of motion existing under the resonance conditions (2). It is easy to check that the dependent condition (3) does not alter the scenario of Fig. 2c (although this is not a general rule, as discussed in [1]); it therefore represents all the 
classes of motion existing up order $K=3$. In summary, there are eight classes of motion: two monomodal solutions $\left(\left\{A_{1}\right\}\right.$ and $\left.\left\{A_{5}\right\}\right)$, three bi-modal solutions $\left(\left\{A_{1}, A_{2}\right\}, \quad\left\{A_{1}, A_{5}\right\}\right.$ and $\left.\left\{A_{3}, A_{4}\right\}\right)$, one three-modal $\left(\left\{A_{1}, A_{3}, A_{4}\right\}\right)$, one four-modal $\left(\left\{A_{1}, A_{2}, A_{3}, A_{4}\right\}\right)$ and the complete solution $\left(\left\{A_{1}, A_{2}, A_{3}, A_{4}, A_{5}\right\}\right)$, as already found in [1] by applying the RCM. It is also seen that $A_{1}$ is the most important amplitude, since it participates in six of eight classes. In contrast $A_{5}$ is a less important amplitude, since it contributes to only three classes. This circumstance suggests introducing the concept of the participation index of a given amplitude, i.e., the ratio between the number of classes in which the amplitude appears and the total number of classes existing. From a geometrical point of view it is equal to the ratio between the number of closed curves surrounding that amplitude in the family graph and the number of all the closed curves. Thus the index of $A_{1}$ is $3 / 4$, that of $A_{3}$ and $A_{4}$ is $1 / 2$, that of $A_{2}$ and $A_{5}$ is $3 / 8$. The participation index establishes a hierarchic order among the amplitudes involved in the resonant motion. The higher the participation index, the higher the contribution of the amplitude. If the participation index is equal to 1 , the amplitude participates in all the motions.

As a second (and third) example the system considered above is taken, by adding an external resonance to the internal resonances (2), namely,

$\omega_{0}=\omega_{1} \quad$ or $\quad \omega_{0}=2 \omega_{1}+\omega_{5}$,

entailing the dependent conditions $\omega_{0}=2 \omega_{2}, \omega_{2}=\omega_{4}+2 \omega_{5}, \omega_{2}=\omega_{1} \pm 2 \omega_{3} \mp \omega_{4}$, respectively. However, as in the previous example, it can be seen that they do not affect the classes of motion. A new space $\mathscr{S}_{0}=\left\{A_{0}, A_{1}\right\}$ or $\mathscr{S}_{0}=\left\{A_{0}, A_{1}, A_{5}\right\}$ must be added to those in Fig. 2a. The relevant $\mathscr{F}_{0}$ elementary families drawn from Table 2 are plotted in Fig. $3 \mathrm{a}_{1}$ and $3 \mathrm{a}_{2}$. To build up the multiple classes of motion, $\mathscr{F}_{0123}$, the interaction between the $\mathscr{F}_{123}$ (drawn in Fig. 2c) and $\mathscr{F}_{0}$ families must be analyzed. Resonance (4.1) is first studied. Since the forcing amplitude $A_{0}$ cannot vanish, it must be contained in every multiple class (see Corollary 2); the same property therefore holds for $A_{1}$, which is connected with $A_{0}$ in $\mathscr{F}_{0}$. Consequently, all the classes of $\mathscr{F}_{123}$ not containing $A_{1}$ are destroyed by the interaction with $\mathscr{F}_{0}$. The scenario in Fig. 2c therefore simplifies in those of Fig. $3 b_{1}$. It should be noted that although the classes $\left\{A_{3}, A_{4}\right\}$ and $\left\{A_{5}\right\}$ would satisfy the conditions of Theorem 1, they are not multiple classes
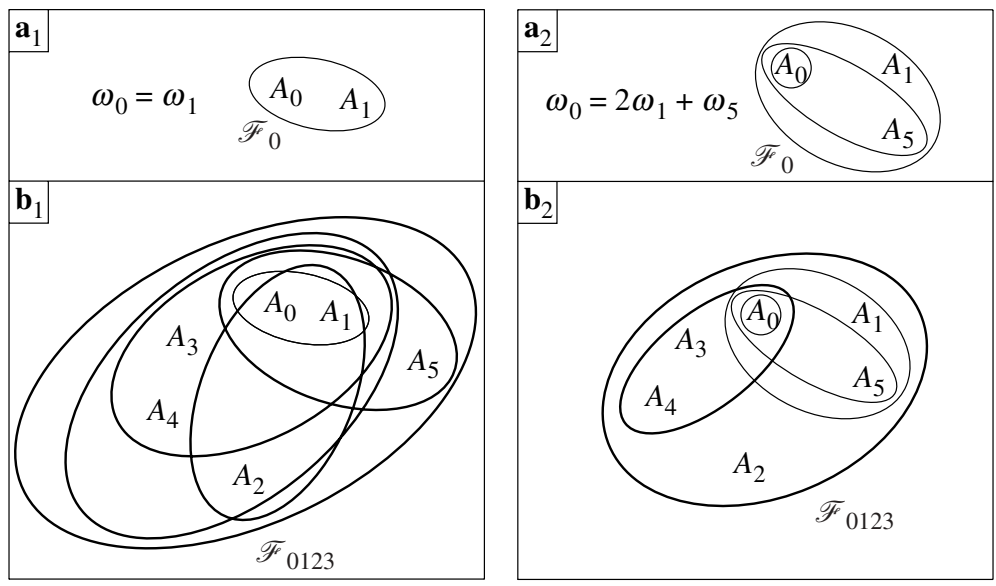

Fig. 3. Class of motion diagram for a system undergoing $R=4$ resonance conditions, the three internal resonances of Fig. 2 plus one external resonance of Fig. $a_{1}$ or Fig. $a_{2}: \mathbf{a}_{1}$, $\mathbf{a}_{2}$ : elementary family $\mathscr{F}_{0}$ of dominion $\mathscr{D}_{0} ; \mathbf{b}_{1}, \mathbf{b}_{2}$ : overall multiple family $\mathscr{F}_{0123}$, resulting from the interaction between the $\mathscr{F}_{123}$ family of Fig. 2 c and the $\mathscr{F}_{0}$ family of Fig. $a_{1}$ or Fig. $a_{2}$, respectively 
because of Corollary 2 . To sum up, in the $\omega_{0}=\omega_{1}$ case six classes still exist, since the excitation only inhibits the bi-modal $\left\{A_{3}, A_{4}\right\}$ and monomodal $\left\{A_{5}\right\}$ solutions of $\mathscr{F}_{123}$. The participation index of amplitude $A_{1}$ is 1 , that of $A_{2}, A_{3}$ and $A_{4}$ is $1 / 2$, and that of $A_{5}$ is $1 / 3$.

Resonance (4.2) is then analyzed. The $\mathscr{F}_{0}$-family associated with Eq. (4.2) is drawn in Fig. $3 \mathrm{a}_{2}$, as furnished by Table 3 . Unlike the example in Fig. $3 \mathrm{a}_{1}$ it admits the incomplete $\left\{A_{0}, A_{5}\right\}$ class of motion. From Corollary 1 all the classes of $\mathscr{F}_{123}$ not containing $A_{5}$ are disrupted, so that only $\left\{A_{0}, A_{5}\right\}$ and $\left\{A_{0}, A_{1}, A_{5}\right\}$ (drawn in thin lines in Fig. 3b) survive. The possible merging among classes is then analyzed. Class $\left\{A_{1}, A_{2}\right\}$ cannot merge with classes of $\mathscr{F}_{0}$, since $\left\{A_{1}, A_{2}, A_{5}\right\}$ is not a class of $\mathscr{F}_{123}$. For similar reasons $\left\{A_{1}, A_{3}, A_{4}\right\}$ cannot merge. However, $\left\{A_{3}, A_{4}\right\}$ merges with both $\left\{A_{0}, A_{5}\right\}$ and $\left\{A_{0}, A_{1}, A_{5}\right\}$. The following classes therefore exist in addition to the complete classes: $\left\{A_{0}, A_{5}\right\},\left\{A_{0}, A_{1}, A_{5}\right\},\left\{A_{0}, A_{1}, A_{3}, A_{4}, A_{5}\right\}$. Moreover, $A_{5}$ has a participation index of $3 / 5, A_{1}, A_{3}$ and $A_{4}$ have an index of $2 / 5$ and $A_{2}$ has $1 / 5$.

\subsection{Existence of standard form AMEs}

The authors discussed in a previous paper [2] how more forms exist to express the complex AMEs in real quantities, each exhibiting some peculiarities with respect to the others. In particular, it is shown in [2] that the most convenient form of AMEs is a so-called standard normal form, in which, by using a suitable mixed representation (i.e., polar and Cartesian) for the complex amplitudes $A_{n}$ and eliminating the phases from the set of the equations, a reduced set of equations of the type $\mathbf{y}^{\prime}=\mathbf{f}(\mathbf{y}, t)$ is obtained. The standard normal form permits analysis of both the periodic solutions and their stability, even if the solutions are incomplete. In contrast, the usual polar representation cannot be employed in the stability analysis of such solutions, but Cartesian components must be used and a change of variables introduced to render the equations autonomous. However, in order to obtain the standard form equation, it is necessary that a fundamental hypothesis be satisfied, namely that there exists a set $L:=N-R$ of so-called principal amplitudes (in addition to $A_{0}$ ) that are different from zero in any class of motion admitted by the system. If this hypothesis is not satisfied, the standard normal form equations are valid only for the particular classes of motion in which the principal amplitudes do not vanish.

The geometrical method developed here permits us to detect the existence of standard form AMEs before writing them. To this end, it must be checked that $L$ amplitudes participate in all steady motions, i.e., they have a participation index equal to 1 . For example, for the system in Fig. 2, since $N=5$ and $R=3$ it is necessary to find $L=2$ non-vanishing principal amplitudes. However, no such amplitudes exist, hence, no unique standard form can be built up for that system. However, if $A_{1}$ and $A_{3}$ (or $A_{4}$ ) are taken as principal amplitudes, the relevant standard form equations enable us to analyze the stability of five of the seven classes of motion. If the systems in Fig. 3 are instead considered, since $N=5$ and $R=4$, it is necessary to find only $L=1$ non-vanishing principal amplitudes. One such amplitude (namely $A_{1}$ ) does exist in the resonance $\omega_{0}=\omega_{1}$, but does not exist in the $\omega_{0}=2 \omega_{1}+\omega_{5}$ case.

\section{Classes of perturbation}

The stability problem of steady (periodic or bi-periodic) solutions is addressed. The structure of the Jacobian variational matrix is analyzed using geometrical arguments; illustrative examples are then given. 


\subsection{Jacobian variational matrix}

During a periodic motion the amplitudes $A_{n}$ vary in time as

$A_{n}=\hat{A_{n}} \exp \left(i v_{n} t\right)$,

where $\hat{A_{n}}$ are constant amplitudes and $v_{n}$ are frequency corrections, which exactly tune the nearly-resonant linear frequencies. Similarly, in a periodically modulated (bi-periodic) motion, the $A_{n}$ 's still vary as in Eq. (5), $\hat{A_{n}}$ now being periodic functions. By performing the change of variable (5) in the equations of motion, it can be shown [2]-[4] that equations formally similar to Eqs. (7) of Part I are obtained, in which the time-harmonic dependence of $\mathscr{L}_{m}$ due to the detunings is removed. By collecting the linear terms, these equations read:

$A_{m}^{\prime}-\xi_{m} A_{m}=\hat{\mathscr{L}}_{m}\left(A_{m}\left(A_{n} \overline{A_{n}}\right), \ldots, \prod_{n \in \mathcal{N}^{ \pm}} A_{n}^{l_{s m n}}, \ldots\right), \quad m=1,2, \ldots, N$,

where $\xi_{m}$ are constants, $\hat{\mathscr{L}}_{m}$ is a constant linear operator and the hat has been dropped from the amplitudes $\hat{A}$ to simplify the notation. By making the variation in Eqs. (6), the following variational equations are obtained:

$\delta A_{m}^{\prime}=\sum_{\substack{n \in \mathcal{N}^{ \pm} \\ n / 0}} J_{m n} \delta A_{m}, \quad m=1,2, \ldots, N$,

with $J_{m n}=\xi_{m}+\partial \hat{\mathscr{L}}_{m}\left(\mathscr{S}^{0}\right) / \partial A_{n}$ evaluated at the steady solution $\mathscr{S}^{0}$. Equations (7) are amenable to analyze the stability of periodic (or bi-periodic) solutions, since they are a set of linear equations with constant (or periodic) coefficients.

The geometrical theory previously developed permits us to gain insight into the structure of the Jacobian matrix $\mathbf{J}=\left[J_{m n}\right]$, by simply examining the diagram of the classes of motion. To this end, let us denote by $\mathbf{X}=\left\{X_{i}\right\}, \mathbf{Y}=\left\{Y_{j}\right\}$ and $\mathbf{Z}=\left\{Z_{k}\right\}$ three subsets of the state-space $\mathscr{S}=\left\{A_{n}\right\}=\{\mathbf{X}, \mathbf{Y}, \mathbf{Z}\}$, having the following properties: $\mathbf{X}$ is the set of the (active) amplitudes participating in the steady motion $\mathscr{S}^{0}:=\left\{\mathbf{X}^{0}, \mathbf{0}, \mathbf{0}\right\}$, whose stability is under analysis; $\mathbf{Y}$ is the set of (passive resonant) amplitudes present in all the elementary spaces $\mathscr{S}_{s}$ which contain at least one amplitude $X_{i} ; \mathbf{Z}$ is the set of all the (passive non-resonant) remaining amplitudes. The following Theorem, proved in Appendix B, holds:

Theorem 2: The variational equation (7) based on the steady solution $\mathscr{S}^{0}=\left\{\mathbf{X}^{0}, \mathbf{0}, \mathbf{0}\right\}$ is uncoupled in the variables $\delta \mathbf{X}, \delta \mathbf{Y}$ and $\delta \mathbf{Z}$, namely,

$\delta \mathbf{X}^{\prime}=\mathbf{J}_{\mathbf{X}} \delta \mathbf{X}, \quad \delta \mathbf{Y}^{\prime}=\mathbf{J}_{\mathbf{Y}} \delta \mathbf{Y}, \quad \delta \mathbf{Z}^{\prime}=\mathbf{J}_{\mathbf{Z}} \delta \mathbf{Z}$,

with $\mathbf{J}_{\mathbf{Z}}$ diagonal.

Equations (8) have the following mechanical interpretation. There exist three different classes of perturbation, each of which is independent and which possibly lead $\mathscr{S}^{0}$ to become unstable, namely: X-perturbations, or in-class perturbations; Y-perturbations, or out-of-class resonant perturbations; Z-perturbations, or out-of-class non-resonant perturbations. The three perturbations describe different forms of instability. If $\mathbf{J}_{\mathbf{X}}$ has at least one unstable eigenvalue (or Floquet multiplier), the perturbed motion evolves (at least initially) in the same class $\{\mathbf{X} \neq \mathbf{0}, \mathbf{Y}=\mathbf{Z}=\mathbf{0}\}$. If $\mathbf{J}_{\mathbf{Y}}$ is instead unstable, the perturbed motion leaves the class, since the amplitudes $\mathbf{Y}$, directly involved in the resonances with $\mathbf{X}$, are triggered. Finally, if the eigenvalues of $\mathbf{J}_{\mathbf{Z}}$ are unstable, the non-resonant perturbations $\mathbf{Z}$ grow in time. This last mechanism of instability is due to a combined effect of the eigenvalues $\xi_{k}$ (governing the stability of the trivial solution) and of the improper resonant terms [2] which couple the $\mathbf{X}$ - and $\mathbf{Z}$-amplitudes, as explained in Appendix B. 
A deeper analysis of the structure of the variational equations reveals some additional interesting aspects. It has been observed in the literature [5] that often the Jacobian matrix $\mathbf{J}_{\mathbf{Y}}$ is composed of diagonal blocks, i.e., there exists some uncoupling among the out-of-class resonant perturbations $\delta \mathbf{Y}$. General rules about such uncouplings are drawn directly by the geometrical method illustrated, according to the following Theorem, proven in Appendix B. The Theorem refers to the generic case in which all terms in the equations are assumed not to be zero. If some terms vanish, further uncouplings can be present.

Theorem 3: Given two subsets $\mathbf{Y}_{1}$ and $\mathbf{Y}_{2}$ of $\mathbf{Y}$, such that $\mathbf{Y}=\left\{\mathbf{Y}_{1}, \mathbf{Y}_{2}\right\}$, the perturbations $\delta \mathbf{Y}_{1}$ and $\delta \mathbf{Y}_{2}$ are uncoupled in the variational equation (8.2) if and only if there exists a multiple class $\mathscr{C}^{*}$ containing all the amplitudes $\mathbf{X}$ and the subset $\mathbf{Y}_{1}$.

Theorem 3 has the following consequence: if the smallest class $\mathscr{C}^{*}$ that contains $\mathbf{X}$ and at least one amplitude $Y_{j}$ also contains all the amplitudes $\mathbf{Y}$, then the Jacobian matrix $\mathbf{J}_{\mathbf{Y}}$ is full. In contrast, if such a class contains only some of the $\mathbf{Y}$, then the matrix $\mathbf{J}_{\mathbf{Y}}$ is block-diagonal. The examples in Fig. 4 illustrate four typical cases. In case (a) the Jacobian matrix is full, since no class exists containing $\mathbf{X}$ and a sub-set of $\mathbf{Y}$. In case (b), by applying Theorem 3 to class $\mathscr{C}_{1}^{*}$, it turns out that $\mathbf{J}_{\mathbf{Y}}$ contains a block $\mathbf{J}_{\mathbf{Y}_{1}}$ associated with the variables $\delta \mathbf{Y}_{1}$; the remaining variables are coupled (among them), so that $\mathbf{J}_{\mathbf{Y}}$ is composed of two blocks. In case (c), Theorem 3 can be applied to both classes $\mathscr{C}_{1}^{*}$ and $\mathscr{C}_{2}^{*}$, and again two blocks are found. Case (d) is less straightforward. By applying Theorem 3 to class $\mathscr{C}_{1}^{*}$ it follows that the variables $\delta \mathbf{Y}_{3}$ are uncoupled from $\left\{\delta \mathbf{Y}_{1}, \delta \mathbf{Y}_{2}\right\}$; however, by applying Theorem 3 to class $\mathscr{C}_{2}^{*}$ it follows that $\delta \mathbf{Y}_{1}$ are uncoupled from $\left\{\delta \mathbf{Y}_{2}, \delta \mathbf{Y}_{3}\right\}$. Consequently $\left\{\delta \mathbf{Y}_{1}\right\},\left\{\delta \mathbf{Y}_{2}\right\}$ and $\left\{\delta \mathbf{Y}_{3}\right\}$ are uncoupled from one another, and the Jacobian matrix $\mathbf{J}_{\mathbf{Y}}$ is composed of three blocks. This last example suggests the following Corollary 3 to Theorem 3:

Corollary 3: If there exist $H$ classes $\mathscr{C}_{h}^{*}(h=1,2, \ldots, H)$, each containing $\mathbf{X}$ and a distinct sub-set $\mathbf{Y}_{h}(h=1,2, \ldots, H)$ of $\mathbf{Y}$, then the Jacobian matrix $\mathbf{J}_{\mathbf{Y}}$ is block-diagonal,

$\mathbf{J}_{\mathbf{Y}}=\operatorname{diag}\left[\begin{array}{llll}\mathbf{J}_{\mathbf{Y}_{1}} & \mathbf{J}_{\mathbf{Y}_{2}} & \ldots & \mathbf{J}_{\mathbf{Y}_{B}}\end{array}\right]$

with the number of blocks $B \geq H$.

The examples in Fig. 4 are self-explanatory.

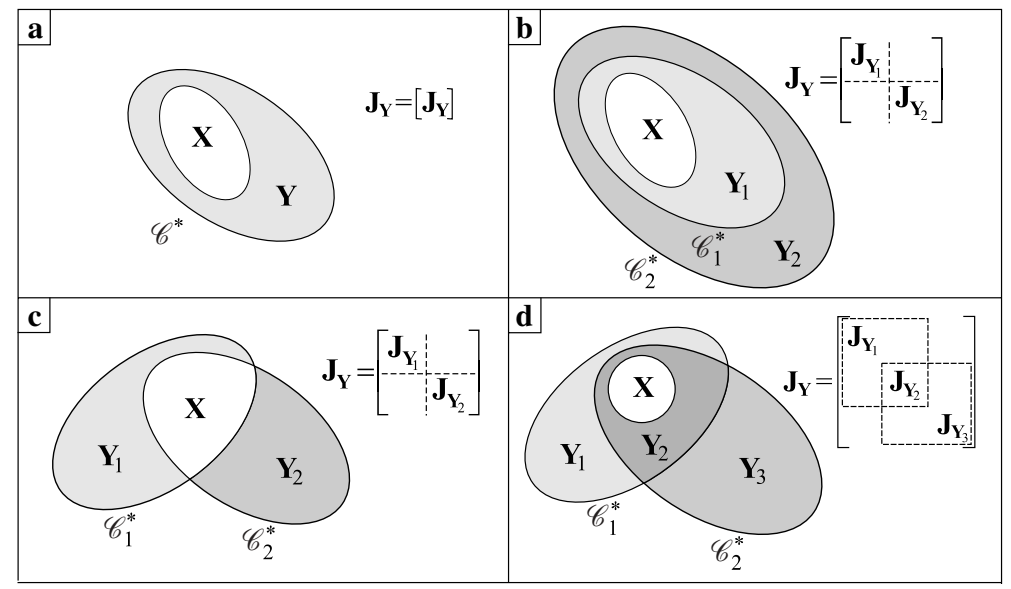

Fig. 4. Classes of motion $\mathscr{C}_{s}^{*}$ containing $\mathbf{X}$ and subsets of $\mathbf{Y}$; structure of the relevant Jacobian matrix $\mathbf{J}_{\mathbf{Y}}$ 
To illustrate some applications of Theorems 2 and 3 let us consider the diagram in Fig. 2c. First, let us analyze the stability of a steady motion $\mathbf{X}=\left\{A_{1}, A_{2}\right\}$. From the elementary resonance spaces in Fig. 2a, it follows that $\mathbf{Y}=\left\{A_{3}, A_{4}, A_{5}\right\}$ (since $A_{3}$ and $A_{5}$ are in resonance with $A_{2}$ in Eq. (2.2), $A_{4}$ in Eq. (3) and $\mathbf{Z}=\{0\}$. Theorem 2 states that the variational equations (7) uncouple in the perturbations $\left\{\delta A_{1}, \delta A_{2}\right\}$ and $\left\{\delta A_{3}, \delta A_{4}, \delta A_{5}\right\}$. Moreover, Theorem 3 states that, since a class $\mathscr{C}_{1}^{*}$ exists (see Fig. 2c), containing $\left\{A_{1}, A_{2}\right\}$ and $\mathbf{Y}_{1}=\left\{A_{3}, A_{4}\right\}$ (i.e., the case of Fig. $4 \mathrm{~b}$ occurs), the variational equations in $\left\{\delta A_{3}, \delta A_{4}, \delta A_{5}\right\}$ are uncoupled in two blocks $\left\{\delta A_{3}, \delta A_{4}\right\}$ and $\left\{\delta A_{5}\right\}$.

As a second example, the stability of a steady solution $\mathbf{X}=\left\{A_{1}, A_{5}\right\}$ is analyzed. From the elementary classes of resonance (2) and (3) it follows that $\mathbf{Y}=\left\{A_{2}, A_{3}\right\}, \mathbf{Z}=\{0\}$. Therefore, according to Theorem 2, $\left\{\delta A_{1}, \delta A_{5}\right\}$ and $\left\{\delta A_{2}, \delta A_{3}, \delta A_{4}\right\}$ are uncoupled in the variational equation. Moreover, since the only class that contains $\left\{A_{1}, A_{5}\right\}$ also contains $\left\{A_{2}, A_{3}, A_{4}\right\}$, the perturbations $\delta A_{2}, \delta A_{3}$ and $\delta A_{4}$ are coupled in the variational equation in accordance with Theorem 3 (i.e., the case of Fig. 4 a occurs).

Finally, as a third example of Fig. 2c, if $\mathbf{X}=\left\{A_{3}, A_{4}\right\}$, then $\mathbf{Y}=\left\{A_{2}, A_{5}\right\}, \mathbf{Z}=\left\{A_{1}\right\}$; since there exists a class $\mathscr{C}_{1}^{*}$ containing $\left\{A_{3}, A_{4}\right\}$ and $\mathbf{Y}_{1}=\left\{A_{2}\right\}$, it follows that $\mathbf{J}_{\mathbf{Y}}$ uncouple in two blocks (Theorem 3).

\section{Conclusions}

A geometrical approach to the problem of evaluating the classes of motion in general multiresonant systems has been illustrated. A method based on a set representation of the classes of motion has been developed, consisting in the following two steps: (a) the set of the classes of motion admitted by each individual resonance (family) is first built up (e.g., by drawing from the tables given here); (b) the interactions among families are studied recursively in pairs, by applying simple rules generated by a unique theorem. The method furnishes class diagrams which efficiently synthesize the couplings existing among the amplitudes as well as their importance in describing the motion. In particular, by using these diagrams it is possible to ascertain in advance whether or not the Amplitude Modulation Equations (AMEs) governing the asymptotic behavior of the system admit a standard form, according to the findings of a previous work [2].

The structure of the variational equations governing the stability of steady (periodic or biperiodic) solutions has then been analyzed. Three types of perturbation have been identified: (a) in-class perturbations, i.e., perturbations of the amplitudes participating in the motion; (b) nonresonant, and (c) resonant out-of-class perturbations, i.e., perturbations of the amplitudes not participating in the motion directly or not involved in resonance with the active amplitudes, respectively. It has been proved that the Jacobian matrix is block diagonal and does not contain coupling terms among the three classes of perturbations. Finally, based on the class diagrams, it is also possible to gain information on possible further uncouplings of the Jacobian block associated with the resonant out-of-class perturbations. It has been found that improper resonant terms which are unessential to the class evaluation do nonetheless contribute to the stability of steady motion. 


\section{Appendix A}

\section{Proof of Theorem 1}

Using the symbols in Fig. 1, the equations governing the modulation of the amplitudes of the space $\mathscr{A}$ are of the type

$\mathbf{U}^{\prime}=\mathbf{f}(\mathbf{U}, \mathbf{W}), \quad \mathbf{W}^{\prime}=\mathbf{h}(\mathbf{U}, \mathbf{W})$,

and those of the space $\mathscr{B}$ are

$\mathbf{V}^{\prime}=\mathbf{g}(\mathbf{V}, \mathbf{W}), \quad \mathbf{W}^{\prime}=\mathbf{k}(\mathbf{V}, \mathbf{W})$.

In Eqs. (A.1) and (A.2) only primary resonant terms (governing the existence of the classes) should be retained [1]. When the two resonances act simultaneously, the equations assemble as follows [1]:

$\mathbf{U}^{\prime}=\mathbf{f}(\mathbf{U}, \mathbf{W}), \quad \mathbf{V}^{\prime}=\mathbf{g}(\mathbf{V}, \mathbf{W}), \quad \mathbf{W}^{\prime}=\mathbf{h}(\mathbf{U}, \mathbf{W})+\mathbf{k}(\mathbf{V}, \mathbf{W})$.

Let $\mathscr{C}=\{\widehat{\mathbf{U}}, \widehat{\mathbf{V}}, \widehat{\mathbf{W}}\}$ be a set of amplitudes, with $\mathbf{U}=\left\{\mathbf{U}_{0}, \widehat{\mathbf{U}}\right\}, \mathbf{V}=\left\{\mathbf{V}_{0}, \widehat{\mathbf{V}}\right\}, \mathbf{W}=\left\{\mathbf{W}_{0}, \widehat{\mathbf{W}}\right\}$. After having partitioned, Eqs. (A.3) read:

$$
\begin{aligned}
& \mathbf{U}_{0}^{\prime}=\mathbf{f}_{0}\left(\mathbf{U}_{0}, \mathbf{W}_{0} ; \widehat{\mathbf{U}}, \widehat{\mathbf{W}}\right), \hat{\mathbf{U}}^{\prime}=\hat{\mathbf{f}}\left(\mathbf{U}_{0}, \mathbf{W}_{0} ; \widehat{\mathbf{U}}, \widehat{\mathbf{W}}\right), \\
& \mathbf{V}_{0}^{\prime}=\mathbf{g}_{0}\left(\mathbf{V}_{0}, \mathbf{W}_{0} ; \widehat{\mathbf{V}}, \widehat{\mathbf{W}}\right), \hat{\mathbf{V}}^{\prime}=\hat{\mathbf{g}}\left(\mathbf{V}_{0}, \mathbf{W}_{0} ; \widehat{\mathbf{V}}, \widehat{\mathbf{W}}\right), \\
& \mathbf{W}_{0}^{\prime}=\mathbf{h}_{0}\left(\mathbf{U}_{0}, \mathbf{W}_{0} ; \widehat{\mathbf{U}}, \widehat{\mathbf{W}}\right)+\mathbf{k}_{0}\left(\mathbf{V}_{0}, \mathbf{W}_{0} ; \widehat{\mathbf{V}}, \widehat{\mathbf{W}}\right), \\
& \hat{\mathbf{W}}^{\prime}=\hat{\mathbf{h}}\left(\mathbf{U}_{0}, \mathbf{W}_{0} ; \widehat{\mathbf{U}}, \widehat{\mathbf{W}}\right)+\hat{\mathbf{k}}\left(\mathbf{V}_{0}, \mathbf{W}_{0} ; \widehat{\mathbf{V}}, \widehat{\mathbf{W}}\right) .
\end{aligned}
$$

If $\mathscr{C}$ is a class of motion of the multiple resonance, then $\mathbf{f}_{0}(\mathbf{0}, \mathbf{0} ; \widehat{\mathbf{U}}, \widehat{\mathbf{W}})=\mathbf{0}, \mathbf{g}_{0}(\mathbf{0}, \mathbf{0} ; \widehat{\mathbf{V}}, \widehat{\mathbf{W}})=\mathbf{0}$, $\mathbf{h}_{0}(\mathbf{0}, \mathbf{0} ; \widehat{\mathbf{U}}, \widehat{\mathbf{W}})+\mathbf{k}_{0}(\mathbf{0}, \mathbf{0} ; \widehat{\mathbf{V}}, \widehat{\mathbf{W}})=\mathbf{0} \forall(\widehat{\mathbf{U}}, \widehat{\mathbf{V}}, \widehat{\mathbf{W}})$. Since $\mathbf{h}_{0}$ and $\mathbf{k}_{0}$ depend on different variables, $\mathbf{h}_{0}(\cdot)=\mathbf{0}, \mathbf{k}_{0}(\cdot)=\mathbf{0}$ follows. Therefore $\mathscr{C} \cap \mathscr{A}=\{0,0 ; \widehat{\mathbf{U}}, \widehat{\mathbf{W}}\}$ is a class of $\mathscr{A}$ and $\mathscr{C} \cap \mathscr{B}=\{0,0 ; \widehat{\mathbf{V}}, \widehat{\mathbf{W}}\}$ is a class of $\mathscr{B}$. Vice versa, if $\mathscr{C} \cap \mathscr{A}$ and $\mathscr{C} \cap \mathscr{B}$ are elementary classes of $\mathscr{A}$ and $\mathscr{B}$, respectively, then $\mathbf{f}_{0}(\cdot)=\mathbf{0}, \mathbf{h}_{0}(\cdot)=\mathbf{0}$ and $\mathbf{g}_{0}(\cdot)=\mathbf{0}, \mathbf{k}_{0}(\cdot)=\mathbf{0}$ and therefore $\mathscr{C}$ is a multiple class.

\section{Appendix B}

\section{Proofs of Theorems 2 and 3}

Theorems 2 and 3 are proved here. Preliminarily, it is recalled from [1] that in the $n$-th AME terms of the following forms are present: (a) improper resonant terms of the type $A_{n}\left(A_{i} \overline{A_{i}}\right)^{k_{i}}, A_{n}\left(A_{i} \overline{A_{i}}\right)^{k_{i}}\left(A_{j} \overline{A_{j}}\right)^{k_{j}}, \ldots$, with $k_{i}$ and $k_{j}$ integer; (b) primary proper resonant terms of the type $\prod_{i \quad 0}^{N} A_{i}^{l_{i}} \bar{A}_{i}^{l}{ }^{i}$ with $\sum_{i{ }_{0}}^{N}\left(l_{i}-l_{i}\right) \omega_{i}=\omega_{n}$, i.e., terms whose frequency is resonant with the $n$-th frequency; (c) secondary proper resonant terms, obtained from the proper ones by multiplying them by $\left(A_{i} \overline{A_{i}}\right)^{k_{i}},\left(A_{i} \overline{A_{i}}\right)^{k_{i}}\left(A_{j} \overline{A_{j}}\right)^{k_{j}}$. The following notation is adopted here: given a set $\mathbf{X}=\left\{X_{i}\right\} i \in \mathscr{I}$ of amplitudes, the homogeneous polynomial of degree $l$ in the variables $X_{i}$ and $\bar{X}_{i}$ is denoted by $\mathbf{X}^{l}$, i.e.,

$\mathbf{X}^{l}:=\left\{\prod_{i \in \mathscr{I}} X_{i}^{l_{i}} \bar{X}_{i}^{l^{i}}, \quad \forall l_{i} \mid \sum_{i \in \mathscr{I}} l_{i}+l_{i}=l\right\}$. 
The following Lemma holds:

Lemma 1: If $\mathbf{X}=\left\{X_{i}\right\}$ is a multiple class of motion, then Eqs. (6) governing the evolution of $\mathbf{X}$ do not contain terms that are linear in the amplitudes $\mathbf{Y}=\left\{Y_{j}\right\}$ and $\mathbf{Z}=\left\{Z_{k}\right\}$ not belonging to the class.

Proof: First, it is observed that improper resonant terms as well as secondary resonant terms [1] are quadratic in $\mathbf{Y}$ and $\mathbf{Z}$; therefore, only primary resonant terms must be analyzed. If a term that is linear in $\mathbf{Y}$ or $\mathbf{Z}$ (e.g., $\mathbf{Y}_{q}$ ) were present in the $p$-th $\mathbf{X}$-equation, then a resonance condition of the following type would exist:

$\omega_{X_{p}}=\sum_{i \in \mathscr{I}} K_{i} \omega_{X_{i}} \pm \omega_{Y_{q}}$,

in which $\omega_{X}$ and $\omega_{Y}$ would be $\mathbf{X}$ - and $\mathbf{Y}$-frequencies, respectively. Equation (B.2) would entail the $\mathbf{Y}_{q}$-equation containing proper resonant terms that are pure in the amplitudes $\mathbf{X}$; consequently $\mathbf{X} \neq \mathbf{0}$ and $\mathbf{Y}=\mathbf{Z}=\mathbf{0}$ would not be a class of the system, contrary to the hypothesis. Therefore, no linear terms in $\mathbf{Y}$ or $\mathbf{Z}$ can exist in the $\mathbf{X}$-equation.

Proof of Theorem 2: The equations of motion are polynomial in $\mathbf{X}, \mathbf{Y}$ and $\mathbf{Z}$. However, only terms that are pure in $\mathbf{X}$ and linear in $\mathbf{Y}$ or $\mathbf{Z}$ contribute to the variational equation, since their derivatives are evaluated at $\mathbf{X} \neq \mathbf{0}$ and $\mathbf{Y}=\mathbf{Z}=\mathbf{0}$. Therefore the essential parts of the equations of motion read:

$$
\begin{aligned}
& \mathbf{X}^{\prime}-\boldsymbol{\xi} \mathbf{X}=\mathbf{f}\left(\mathbf{X}^{l}, \mathbf{X}^{m} \mathbf{Y}, \mathbf{X}^{m} \mathbf{Z}\right), \\
& \mathbf{Y}^{\prime}-\boldsymbol{\eta} \mathbf{Y}=\mathbf{g}\left(\mathbf{X}^{l}, \mathbf{X}^{m} \mathbf{Y}, \mathbf{X}^{m} \mathbf{Z}\right), \\
& \mathbf{Z}^{\prime}-\boldsymbol{\zeta} \mathbf{Z}=\mathbf{h}\left(\mathbf{X}^{l}, \mathbf{X}^{m} \mathbf{Y}, \mathbf{X}^{m} \mathbf{Z}\right), \quad l=2,3, \ldots, \quad m=1,2, \ldots,
\end{aligned}
$$

where $\xi, \eta, \zeta$ are diagonal matrices. Now, by virtue of Lemma $1, \mathbf{X}^{m} \mathbf{Y}$ and $\mathbf{X}^{m} \mathbf{Z}$ cannot be present in the $\mathbf{X}$-equation; moreover, terms that are pure in $\mathbf{X}$ cannot exist in the $\mathbf{Y}$ - and $\mathbf{Z}$ equations, since $\mathbf{X}$, by hypothesis, is a class of motion. Further, the terms $\mathbf{X}^{m} \mathbf{Z}$ and $\mathbf{X}^{m} \mathbf{Y}$ must vanish in the $\mathbf{Y}$ - and $\mathbf{Z}$-equations, respectively, since (a) they are not improper resonant terms (which would be proportional to $\mathbf{Y}$ and $\mathbf{Z}$, respectively), and (b) they are not proper resonant terms, given that, according to the hypothesis, $\mathbf{X}, \mathbf{Y}$ and $\mathbf{Z}$ are never involved together in a resonance condition. Therefore, Eqs. (B.3) further simplify as:

$\mathbf{X}^{\prime}-\boldsymbol{\xi} \mathbf{X}=\mathbf{f}\left(\mathbf{X}^{l}\right), \quad \mathbf{Y}^{\prime}-\boldsymbol{\eta} \mathbf{Y}=\mathbf{g}\left(\mathbf{X}^{m} \mathbf{Y}\right), \quad \mathbf{Z}^{\prime}-\zeta \mathbf{Z}=\mathbf{h}\left(\mathbf{X}^{m} \mathbf{Z}\right)$.

Terms in the first two equations are proper as well improper resonant terms. Terms in the third equation are only improper resonant terms, since $\mathbf{Z}$ is not involved in resonance with $\mathbf{X}$; therefore, the $k$-th $\mathbf{Z}$-equation contains only the variable $Z_{k}$ out of the set $\mathbf{Z}$. By taking the variation of Eqs. (B.4) and evaluating it at $\mathscr{S}^{0}=\left\{\mathbf{X}^{0}, \mathbf{0}, \mathbf{0}\right\}$, it follows that:

$\delta \mathbf{X}^{\prime}=\left(\boldsymbol{\xi}+\mathbf{f}_{\mathbf{X}}^{0}\right) \delta \mathbf{X}, \quad \delta \mathbf{Y}^{\prime}=\left(\boldsymbol{\eta}+\mathbf{g}_{\mathbf{Y}}^{0}\right) \delta \mathbf{Y}, \quad \delta \mathbf{Z}^{\prime}=\left(\zeta+\mathbf{h}_{\mathbf{Z}}^{0}\right) \delta \mathbf{Z}$,

where $\mathbf{h}_{\mathbf{Z}}^{0}$ is a diagonal matrix. Equations (B.5) prove Theorem 2.

Proof of Theorem 3: The equations of motion (B.3.2) governing the evolution of $\mathbf{Y}=\left\{\mathbf{Y}_{1}, \mathbf{Y}_{2}\right\}^{T}$ from Eq. (B.4.2) are of the type: 


$$
\begin{aligned}
& \mathbf{Y}_{1}^{\prime}-\boldsymbol{\eta}_{1} \mathbf{Y}_{1}=\mathbf{g}_{1}\left(\mathbf{X}^{m} \mathbf{Y}_{1}, \mathbf{X}^{m} \mathbf{Y}_{2}\right) \\
& \mathbf{Y}_{2}^{\prime}-\boldsymbol{\eta}_{2} \mathbf{Y}_{2}=\mathbf{g}_{2}\left(\mathbf{X}^{m} \mathbf{Y}_{1}, \mathbf{X}^{m} \mathbf{Y}_{2}\right) .
\end{aligned}
$$

Let us assume that a class $\mathscr{C}^{*}$ exist, containing all the amplitudes $\mathbf{X}$ and the subset $\mathbf{Y}_{1}$. Then, according to Lemma $1, \mathbf{X}^{m} \mathbf{Y}_{2}$ cannot be present in the first equation; moreover, since $\mathbf{g}_{2}$ must vanish when $\mathbf{Y}_{2}=\mathbf{0}$, terms $\mathbf{X}^{m} \mathbf{Y}_{1}$ cannot be present in $\mathbf{g}_{2}$. By taking the variation of Eq. (B.6) and evaluating it at $\left(\mathbf{X} \neq \mathbf{0}, \mathbf{Y}_{1}=\mathbf{Y}_{2}=\mathbf{0}\right)$, it follows that:

$\delta \mathbf{Y}_{1}^{\prime}=\left(\boldsymbol{\eta}_{1}+\mathbf{g}_{1 \mathbf{Y}_{1}}^{0}\right) \delta \mathbf{Y}_{1}, \quad \delta \mathbf{Y}_{2}^{\prime}=\left(\boldsymbol{\eta}_{2}+\mathbf{g}_{2 \mathbf{Y}_{2}}^{0}\right) \delta \mathbf{Y}_{2}$,

which proves Theorem 3 .

Remark: Unlike $\mathbf{J}_{\mathbf{Y}}$, the Jacobian $\mathbf{J}_{\mathbf{X}}$ is generally a full matrix for the following reasons. The variational equation in $\delta \mathbf{X}=\left\{\delta \mathbf{X}_{1}, \delta \mathbf{X}_{2}\right\}$ is obtained by varying Eq. (B.4.1). Since $\mathbf{X}_{1} \neq \mathbf{0}$ and $\mathbf{X}_{2} \neq \mathbf{0}$, the variational equations in $\delta \mathbf{X}_{1}$ and $\delta \mathbf{X}_{2}$ would be uncoupled if and only if the equations themselves were uncoupled in this term. However, due to the presence of the improper resonant terms this circumstance never occurs in the generic case. Therefore, improper resonant terms, which are unessential to the class evaluation [1], in contrast affect the structure of the variational equation.

\section{Acknowledgement}

This work was supported by MURST (COFIN 01-03).

\section{References}

[1] Luongo, A., Paolone, A., Di Egidio, A.: Qualitative analysis of classes of motion for multiresonant systems I. An algebraic method. Acta Mech. 174, 91-107 (2005).

[2] Luongo, A., Di Egidio, A., Paolone, A.: On the proper form of the amplitude modulation equations for resonant system. Nonlinear Dynamics 27, 237-254 (2002).

[3] Crespo da Silva, M. R. M, Zaretzky, C. L.: Nonlinear flexural-torsional interactions in beams including the effect of torsional dynamics I: Primary resonance. Nonlinear Dynamics 5, 3-23 (1994).

[4] Di Egidio, A., Luongo, A., Vestroni, F.: Nonstationary nonplanar free motions of an orbiting string with multiple internal resonances. Meccanica 31, 363-381 (1996).

[5] Luongo, A., Vestroni, F.: Bifurcations and stability of nonstationary resonant planar oscillations of an orbiting string. Nonlinear Dynamics 9, 305-325 (1996).

Authors' addresses: A. Luongo and A. Di Egidio, Dipartimento di Ingegneria delle Strutture, delle Acque e del Terreno, Università di L'Aquila, Monteluco di Roio (L'Aquila) 67040, Italy (E-mail: luongo@ing.univaq.it); A. Paolone, Dipartimento di Ingegneria Strutturale e Geotecnica, Università degli Studi di Roma La Sapienza, Via Eudossiana, 18, Roma 00184, Italy (E-mail: achille.paolone@, uniroma.it) 